\title{
Pore structure analysis of directionally solidified porous copper
}

\author{
Xiao-bang Liu', *Yan-xiang Li ${ }^{1,2}$, Hua-wei Zhang ${ }^{1,2}$, Yuan Liu ${ }^{1,2}$, Xiang Chen ${ }^{1,2}$ \\ 1. School of Materials Science and Engineering, Tsinghua University, Beijing 100084, China \\ 2. Key Laboratory for Advanced Materials Processing Technology, Ministry of Education, Beijing 100084, China
}

\begin{abstract}
Directionally solidified porous copper is considered as a potential candidate in the field of microchannel heat sinks. By the Bridgman-type directional solidification method, a porous copper ingot was fabricated. Evolution of the porosity, pore number density, average pore diameter and average interpore spacing at different ingot heights was investigated. The results show that with the increase of ingot height, the porosity firstly increases and then basically remains unchanged from the ignot height of $65 \mathrm{~mm}$; the pore number density rapidly decreases at first, and the decreasing speed becomes slower when the ignot height higher than $85 \mathrm{~mm}$; the average pore diameter increases and then remains unchanged from the ingot height of $85 \mathrm{~mm}$; the average interpore spacing increases, and the increasing speed of average interpore spacing becomes slower with the increase of height to higher than $85 \mathrm{~mm}$. In order to study the evolution of diameter and spatial distribution of pores, the distribution ranges of pore diameter, nearest-neighbor distance and radial cumulative pore number were analyzed. As the ingot height increases, the distribution ranges of pore diameter and nearest-neighbor distance firstly increase and then tend to be stable. There are no pore clusters and for long distance, the spatial distribution of pores is uniform at different ingot heights. Pore structure and 3D pore morphology of porous copper were observed with the help of light illumination and X-ray tomography. Pore nucleation, pore interruption, pore coalescence, diameter change of pores and lateral displacement of pores were found to exist in the pore structure.
\end{abstract}

Key words: porous metal; directional solidification; pore structure

CLC numbers: TG146.1 $\quad$ Document code: A Article ID: 1672-6421(2020)05-325-07

$\mathrm{D}$ irectionally solidified porous metals have attracted much attention in the past two decades. Since the gas solubility in the solid phase is much less than that in the liquid phase, the solute gas will accumulate ahead of the solidification front. Supersaturation thus induces nucleation of bubbles on the solidification front. Subsequently, pores grow with the solidification of metal. Thus, a type of porous metals in which cylindrical pores are regularly arranged can be obtained ${ }^{[1]}$. This fabrication technology was first developed by Shapovalov and his co-workers ${ }^{[2]}$ and they named this type of porous metals "Gasars", which

\footnotetext{
*Yan-xiang Li

Male, born in 1962, tenure professor in School of Materials Science \& Engineering, Tsinghua University. His research interests concern solidification processing, porous metals (aluminum foams and lotus-type metals), and casting alloys (cast irons, cast steels). He is currently the chairman of the WFO Ferrous Metals Committee and a member of Foundry Institution of Chinese Mechanical Engineering Society.
}

E-mail: yanxiang@tsinghua.edu.cn

Received: 2020-04-28; Accepted: 2020-07-20 was a Russian abbreviation meaning "gas reinforced". Nakajima ${ }^{[3]}$ called them lotus-type porous metals. According to the preparation process, here it is called a directional solidification porous metal. In this technology, hydrogen ${ }^{[4]}$ or nitrogen ${ }^{[5]}$ is usually taken as the dissolving gas. Insoluble argon is often taken as the auxiliary gas to control pore diameter and porosity in a more flexible way. However, due to the mushy zone effect of alloys, the pore morphology becomes more and more irregular with the increase of alloying element content. Thus, Gasar structure is mainly prepared by pure metals.

Porous metals with directional pores are very suitable materials for heat dissipation ${ }^{[6-10]}$. A great amount of heat can be quickly carried away when fluid flows through cylindrical pores. Pore structure has a significant influence on the heat transfer performance of directionally solidified porous metals ${ }^{[8,9]}$. Ideally, all pores should have the same pore diameter and the length of every pore should be infinite, and pores in the matrix should possess a perfect hexagonal distribution ${ }^{[11]}$. However, the actual pore structure departs from ideal. The pore 
length ranges from a few millimeters to tens of centimeters ${ }^{[12,13]}$. The pore diameter is on the order of hundreds of micrometers ${ }^{[14]}$. Shapovalov ${ }^{[12]}$ schematically depicted the pore nucleation, pore interruption, pore coalescence, diameter change, and orientation change of pores in directionally solidified porous metals. Ide et al. ${ }^{[15]}$ mentioned that coarse pores were evolved by coalescing small pores. Drenchev et al. ${ }^{[16]}$ pointed out that pore coalescence was aimed at obtaining a structure of lower energy. These imperfections of pore structure will decrease the heat transfer performance of directionally solidified porous metal heat sinks.

Previous studies mainly focused on the effects of processing parameters, such as the hydrogen pressure, argon pressure, melt temperature, and solidification rate on structural parameters such as porosity and average pore diameter ${ }^{[4,11]}$. For practical purposes, it is highly important to study the above phenomena ${ }^{[8,17]}$. However, there is still a lack of detailed analysis on actual pore structure in directionally solidified porous metals. In the present study, the porosity, pore number density, average pore diameter, average interpore spacing and the diameter and spatial distribution of pores at different ingot heights of a porous copper ingot were analyzed. Pore structure and 3D pore morphology of directionally solidified porous copper were observed with the help of light illumination and $\mathrm{X}$-ray tomography. Thus, the cause of pore structure evolution is analyzed, and the means of pore structure evolution is summarized.

\section{Experimental method}

\subsection{Directionally solidified porous copper ingot}

The porous copper ingot was fabricated using the Bridgman-type directional solidification method. Figures 1(a) and (b) show the schematic drawings of the apparatus for preparing the ingot. As shown in Fig. 1(a), the furnace chamber is divided into two parts by a heat shield. The upper part, called the hot zone, is used to melt the metal and dissolve hydrogen. The lower part, called the cold zone, is used for solidifying the melt and mechanically transferring the ingot. Research on the optimization of pore structure has been introduced in previous works ${ }^{[18,19]}$. For the porous copper ingot with $100 \mathrm{~mm}$ in diameter and $130 \mathrm{~mm}$ in height (weight about $5.65 \mathrm{~kg}$ ), the optimum withdrawing speed was $1 \mathrm{~mm} \cdot \mathrm{s}^{-1}{ }^{[18]}$.
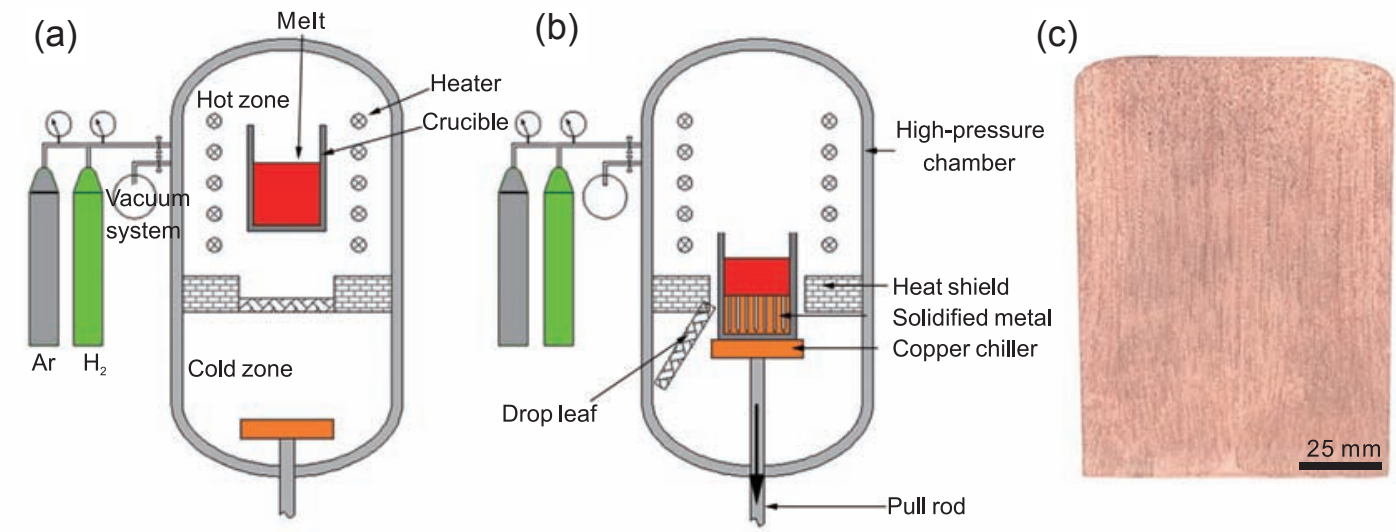

Fig. 1: Schematic drawings of the Bridgman-type directional solidification apparatus: (a) melting and hydrogen dissolving; (b) unidirectional solidification, and (c) longitudinal section of an optimized porous copper ingot

The holding temperature of the melt was $1,407 \mathrm{~K}$ and the holding time was $2 \mathrm{~h}$. The hydrogen and argon pressures were $0.36 \mathrm{MPa}$ and $0.12 \mathrm{MPa}$ respectively. According to the research by Zhang et al. ${ }^{[20]}$, under the degree of superheat of $50 \mathrm{~K}$, a uniform pore structure can be obtained when the partial pressure ratio of hydrogen to argon is about 3:1. Figure 1(c) shows the longitudinal section of the ingot. The porosity of the entire ingot was evaluated through the Archimedes' principle.

\subsection{Characterization methods of pore structure}

First, the cylindrical porous copper ingot was cut into two parts along the central axis with an electric spark wire-cutting machine. Then, one half of the ingot was cut into six pieces perpendicular to the solidification direction at ingot heights of $25,45,65,85,105 \mathrm{~mm}$ from the bottom of the ingot. Each cross-section was polished with a series of abrasive papers from 400 to 2,000 grit. Then, they were degreased with acetone and cleaned with diluted $\mathrm{HCl}$ and ethanol successively in an ultrasonic cleaner. All upper surfaces were scanned by a

\section{HP-G3010 scanner.}

A cuboid sample $(16 \mathrm{~mm} \times 26 \mathrm{~mm} \times 5 \mathrm{~mm})$ was cut from the porous copper ingot at the ingot height of $45-65 \mathrm{~mm}$. A beam of light with enough intensity illuminated the sample perpendicularly from one side to detect the pore structure. The pore structure could be determined based on light information on the other side. Purple paint was used to enhance the contrast to better distinguish the pores and substrate.

Then, a cylindrical sample with a diameter of $4 \mathrm{~mm}$ and a length of $105 \mathrm{~mm}$ was cut from the center of the other half of the ingot. 3D pore morphology of directionally solidified porous copper was obtained by X-ray tomography experiment which was performed with a GE Nanotom CT machine. The $\mathrm{X}$-ray voltage and electric current were chosen as $100 \mathrm{kV}$ and $120 \mu \mathrm{A}$ respectively, and the voxel size was $4 \mu \mathrm{m} \times 4 \mu \mathrm{m} \times 4 \mu \mathrm{m}$. By rotating the sample over $360^{\circ}$, the penetrable intensity information of monochromatic X-ray beam was recorded through a high speed CCD camera. Besides, dark field images and flat field images were also collected for image processing. 


\section{Results and discussion}

\subsection{Evolution of pore structure at different ingot heights}

As shown in Fig. 1(c), the corresponding solid/liquid interface was flat and the ingot porosity was about $38 \%$. Figure 2 shows pore structure on cross-sections at different ingot heights. As shown in Fig. 2, as the ingot height increases, the pore diameter increases and the pore number decreases.

Images of cross-sections were processed by an image analysis system. The centroid coordinate and diameter of each pore were recorded. Figure 3 shows the dependence of porosity, pore number density, average pore diameter and average interpore spacing on the ingot height. As the ingot height increases, the porosity firstly increases and then basically remains unchanged from the ingot height of $65 \mathrm{~mm}$. The pore number density decreases from 665 to 312 per square centimeter when the ingot height increases from $25 \mathrm{~mm}$ to $105 \mathrm{~mm}$, and the decreasing speed of pore number density becomes slower from the ingot height of $85 \mathrm{~mm}$. The average pore diameter increases from $221 \mu \mathrm{m}$ to $358 \mu \mathrm{m}$ and then there is a trend of remaining unchanged from the ingot height of $85 \mathrm{~mm}$. The average interpore spacing increases from $438 \mu \mathrm{m}$ to $656 \mu \mathrm{m}$ when the ingot height increases from $25 \mathrm{~mm}$ to $105 \mathrm{~mm}$, and the increasing speed of average interpore spacing becomes slower from the ingot height of $85 \mathrm{~mm}$.
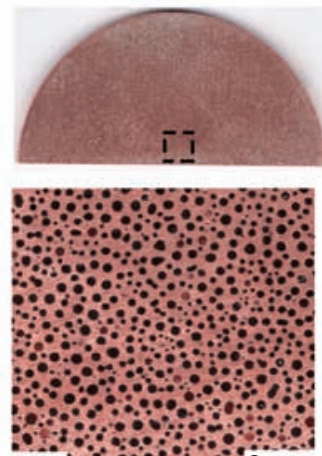

(a) $\ldots 2 \mathrm{~mm}$
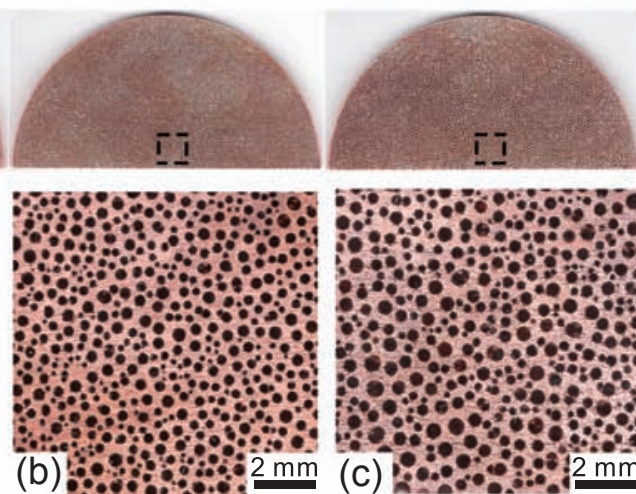

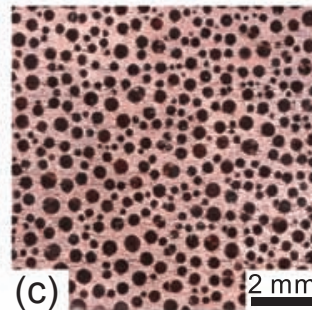

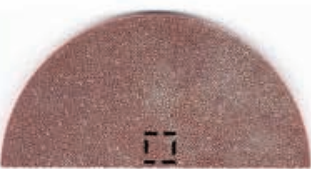
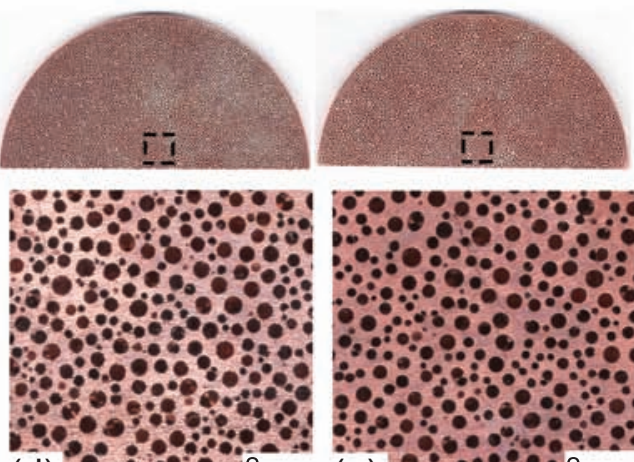

(d) $8: 86: 2 \mathrm{~mm}$

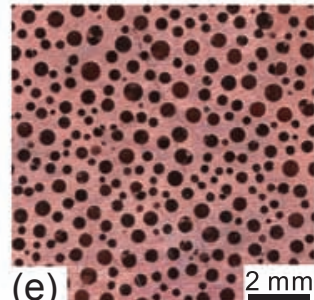

Fig. 2: Pore structure on cross-sections at different ingot heights: (a) $25 \mathrm{~mm}$; (b) $45 \mathrm{~mm}$; (c) $65 \mathrm{~mm}$; (d) 85 mm; (e) $105 \mathrm{~mm}$
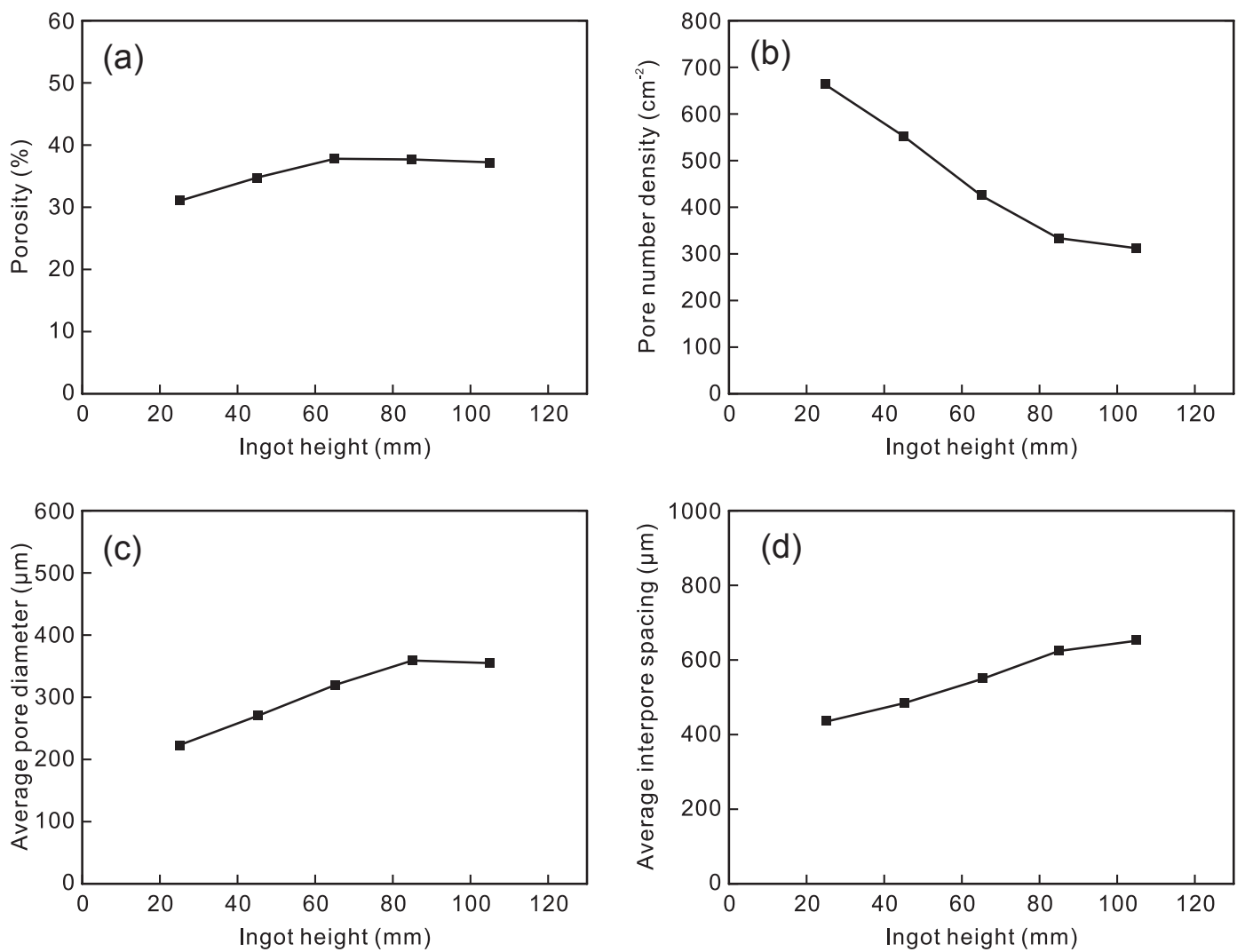

Fig. 3: Evolution of porosity (a), pore number density (b), average pore diameter (c), average interpore spacing (d) along the ingot height 
Diameter and spatial distribution of pores are also essential and important characteristics of porous metals. Figure 4(a) shows the distribution range of pore diameter at different ingot heights. With the increase of ingot height, the distribution range of pore diameter firstly becomes wider and then tends to be stable.

The uniformity of spatial distribution of pores can be reflected by the distribution function of nearest-neighbor distance $^{[21]}$. Statistical results of nearest-neighbor distance at different ingot heights are shown in Fig. 4(b). The nearest- neighbor distance is sensitive to pore clustering ${ }^{[22]}$. If pores are clustered, the function plot of the nearest-neighbor distance shows a peak at a distance close to pore diameter $d$ followed by an abrupt decrease. Thus, based on Fig. 4(a) and Fig. 4(b), there are no pore clusters in the porous copper. Although there is some fluctuation, the spatial distribution of pores is regular. With the increase of ingot height, the average nearest-neighbor distance firstly increases and then remains basically unchanged and its distribution spread firstly becomes wider and then gradually stabilizes.
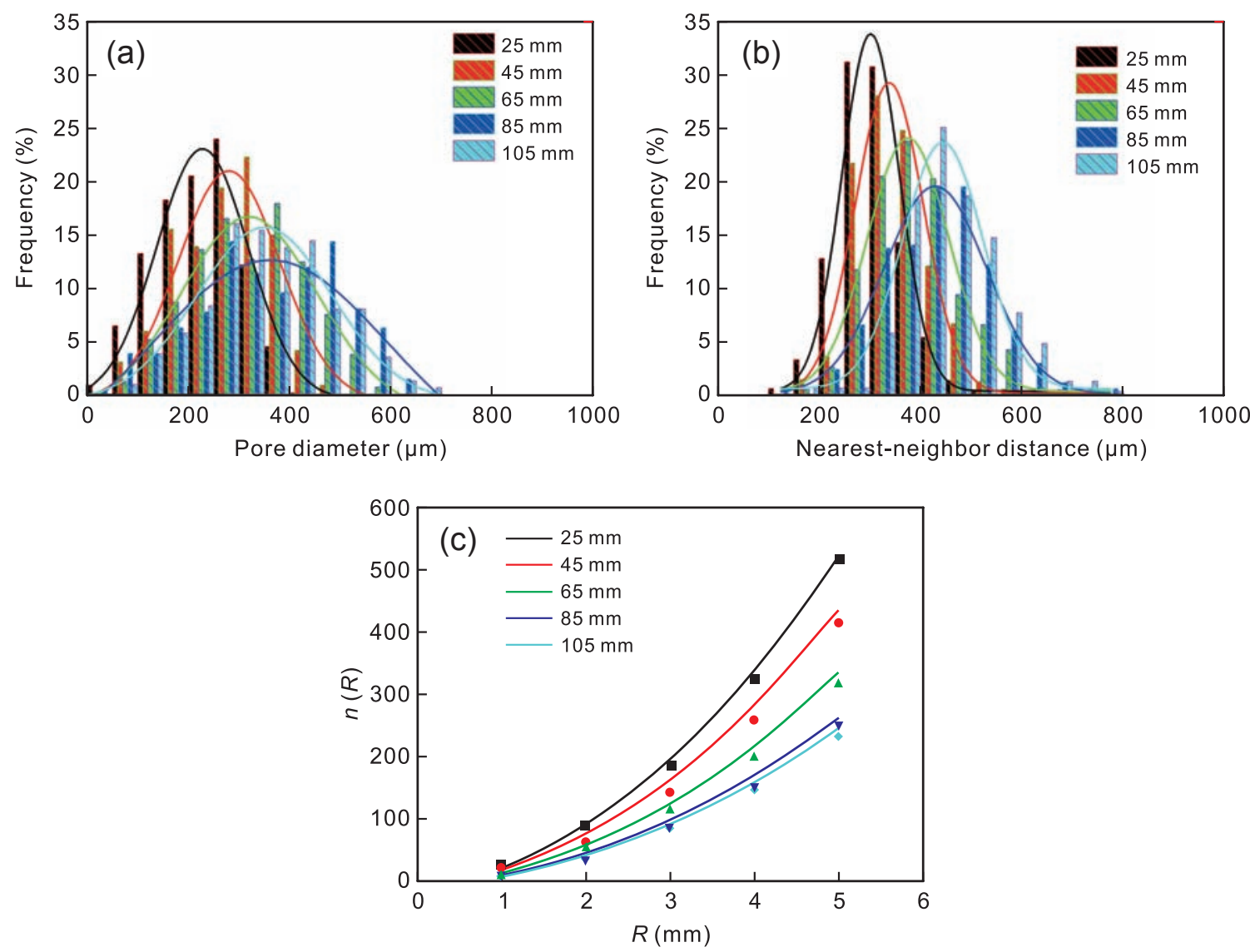

Fig. 4: Evolution of pore diameter distribution (a), nearest-neighbor distance distribution of pores (b), radial cumulative pore number (c) along the ingot height

The uniformity of spatial distribution of pores can also be quantitatively characterized by the radial cumulative pore number $n(R)^{[23]} . n(R)$ is defined as the number of pores which lie within a radial distance $R$ from an arbitrary point. $n(R)$ provides an insight in the arrangement at a range of distances, which is different from the nearest-neighbor distance function. On the condition of uniform spatial distribution, $n(R)$ is not related to the position of the circular region but only directly proportional to its area. It can be analytically evaluated as an ideal form $n^{*}(R)$ :

$$
n^{*}(R)=\pi R^{2} \cdot \frac{N}{A}
$$

where $A$ is the area of the window and $N$ is the total number of pores in the window. Statistical results at different ingot heights are shown in Fig. 4(c). Scatters represent radial cumulative pore number $n(R)$ and lines represent the ideal form of radial cumulative function of pore number $n^{*}(R)$ at different ingot heights. It shows that $n(R)$ agrees well with $n^{*}(R)$. Thus, for long distance, the spatial distribution of pores is uniform at different ingot heights.

\subsection{Cause of pore structure evolution}

Since the pores in directionally solidified porous metals are formed by the hydrogen solubility gap between the liquid and solid phases, the porosity mainly depends on the amount of hydrogen rejected from the solid during directional solidification. The amount of rejected hydrogen is almost constant at the melting point under a constant hydrogen pressure, and thus the porosity should remain unchanged.

However, under a constant gas pressure condition, the pore 
number density, pore diameter and interpore spacing will also be affected by the solidification rate ${ }^{[24]}$. Pore structure evolutions displayed in Fig. 3 and Fig. 4 could be explained by the change of solidification rate.

At the beginning stage of solidification, the solidification front is close to the copper chiller, which results in a great solidification rate. As the solidification height increases, the solidification front is away from the copper chiller, which leads to the decrease of solidification rate.

The solidification rate determines the amount of hydrogen rejected from the solid per unit time. The higher solidification rate, the more hydrogen rejected. According to studies by $\mathrm{He}$ et al. ${ }^{[18]}$ and Jiang et al. ${ }^{[23]}$, with the increase of solidification height, the solidification rate decreases and the slope value of solidification rate decreases. Hence, the hydrogen amount rejected from the solid per unit time decreases, which is insufficient to supply the growth of all pores. Thus, the decrease of solidification rate will aggravate the competitive growth between neighboring pores. It will lead to the decrease of pore number density. As the ingot height increases, the porosity changes little. Ignoring slight changes in porosity, the pore diameter and interpore spacing will increase with a decrease of pore number density. Therefore, the slower the solidification rate, the larger the pore diameter and interpore spacing. It conforms to the variation of lamellar or rod spacing of eutectic growth at varying solidification rate ${ }^{[25]}$.

\subsection{The way of pore structure evolution}

Figure 5 shows pore structure information of a cuboid sample obtained with the help of light illumination. As shown in Fig. 5(a), light illuminated the sample perpendicularly from one side and the scanned picture was obtained from the other side.
Figures 5(b) and (c) show pore structure information observed from the upper surface and lower surface. Figure 5(d) shows the magnified view of specified areas in Figs. 5(b) and (c).

The purple area indicates the copper substrate. White pores indicate that light can pass through these pores, while black pores indicate that light cannot pass through them. Black pores on the upper surface and lower surface convey different information. A black pore on the upper surface indicates that pore nucleation appears in the cuboid sample. However, a black pore on the lower surface indicates that pore interruption appears in the cuboid sample.

Non-circular pores on both the upper and lower surfaces indicate pore coalescence, such as Pores A, B and C. Pore $A$ is located on the upper surface and light can only pass through one branch of the coalescing Pore A. Pore B is located on the lower surface and light cannot pass through the coalescing Pore B. It indicates that Pore B interrupts after pore coalescence. Pore $\mathrm{C}$ is located on the upper surface and light can pass through two branches of the coalescing Pore C. The shadow in Pore $\mathrm{C}$ represents the bubble wall (or ligament) between two pores before coalescence. According to the light information and the surface where the pore is located, the structure of Pores A, B and C inside the cuboid sample can be inferred, as shown in Fig. 5.

There is a crescent-shaped shadow on one side of Pore D, indicating that Pore D has moved laterally during the growth. There is a small inclination angle between the pore growth direction and the solidification direction. In addition, a cascade coalescence of pores is also observed, which means that a pore coalescence is possible to induce the coalescence of surrounding pores. (a)

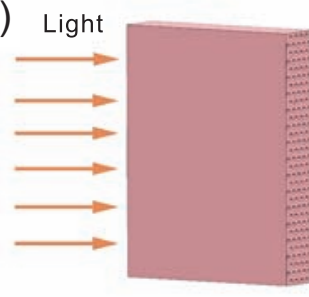

(b)

(d)

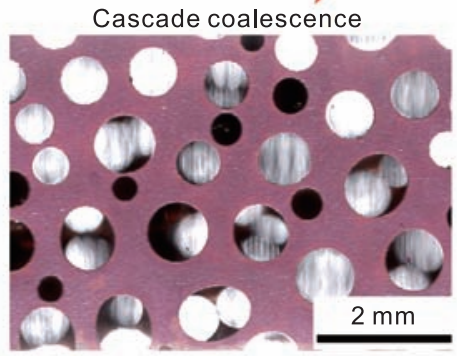

(c)
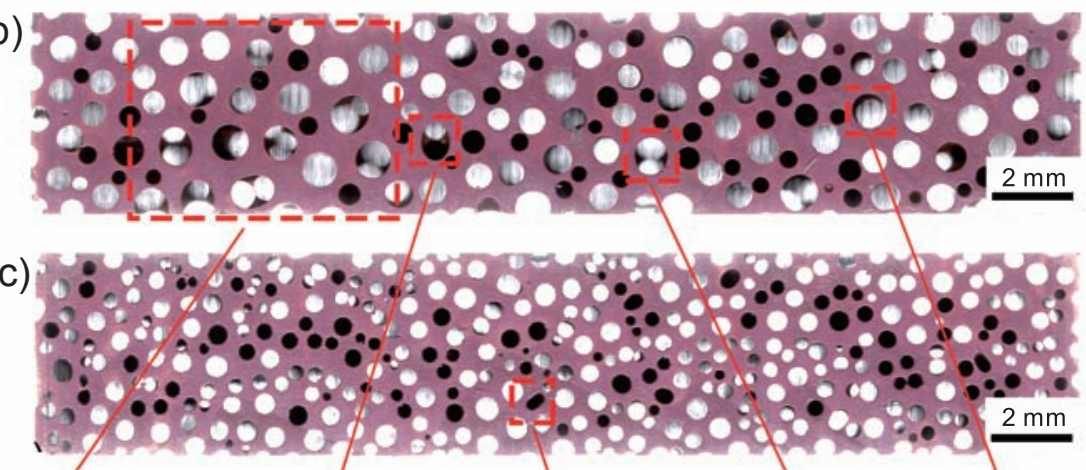

A
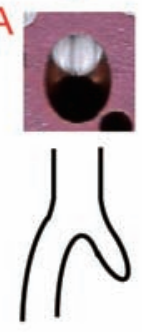

B )
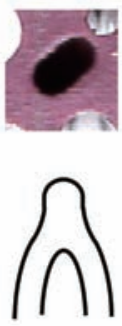

C
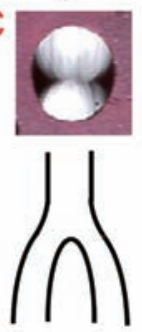

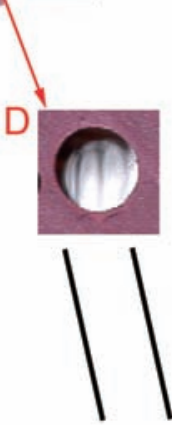

Fig. 5: Pore structure information obtained with the help of light illumination: (a) schematic drawing of light illumination; pore structure information observed from the (b) upper surface and (c) lower surface; (d) magnified view of specified areas 
Figure 6 shows experimental results of X-ray tomography. Figure 6(a) shows a typical 2D image slice from a tomography scan. Figure 6(b) shows the reconstructed 3D pore morphology of directionally solidified porous copper in a domain of $4432 \times 4416 \times 5576(\mu \mathrm{m})$ based on a series of $2 \mathrm{D}$ image slices. Figure $6(\mathrm{c})$ shows the outline extraction of pore structure from the original image. Figure 6(d) shows a continuous superposition of pore structure outlines along the solidification direction. The height interval is $144 \mu \mathrm{m}$. The color changes from red to purple, corresponding to a gradual increase in solidification height. The continuous change of color circles can display the pore growth process.

(a)

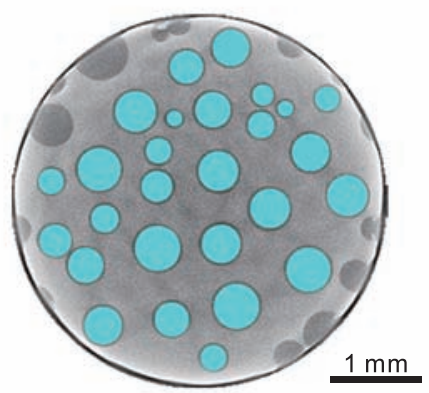

(c)

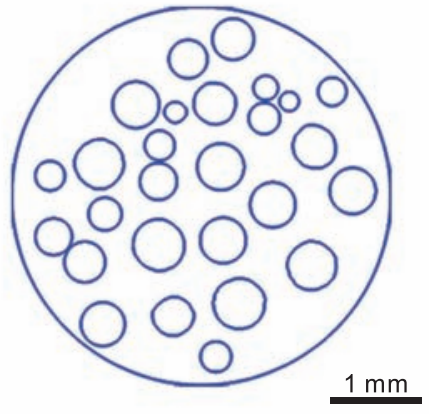

(b)

The nucleation, interruption, coalescence, diameter change, and lateral displacement of pores can be observed in Fig. 6(b) and Fig. 6(d). As shown in Fig. 6(d), Circle A begins to appear in green instead of red, corresponding to pore nucleation. When Circle B turns to orange, its diameter decreases and disappears soon, corresponding to pore interruption. The purple Circle $\mathrm{C}$ results from the coalescence of two small green circles and one of the green circles results from a coalescence of three red circles in the early period. As the color changes from red to purple, the diameter of Circle D increases. As the color changes from red to purple, the centroid of Circle E moves laterally.

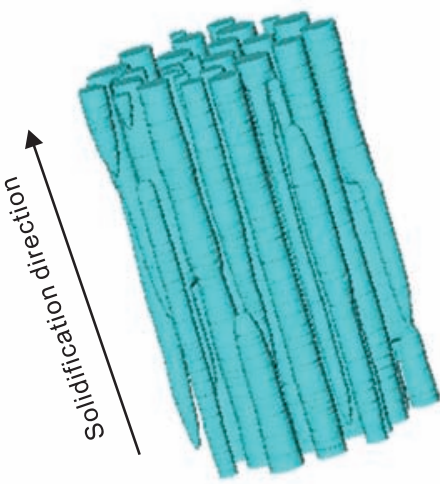

(d)

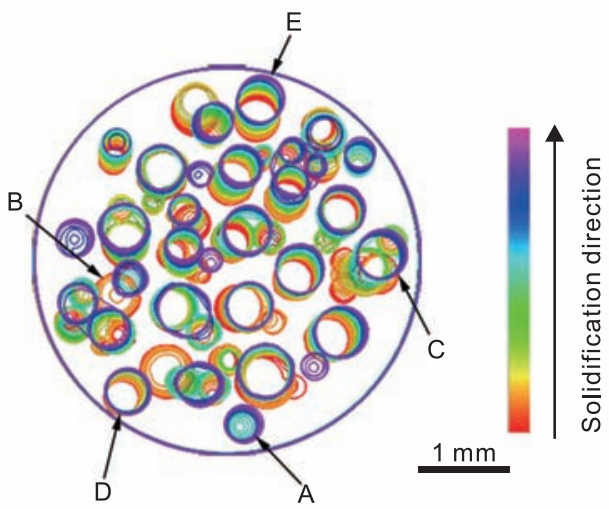

Fig. 6: Experimental results of X-ray tomography: (a) a typical 2D image slice from a tomography scan; (b) reconstructed 3D pore morphology of directionally solidified porous copper; (c) extracting the pore structure outline from the original image; (d) a continuous superposition of pore structure outlines along the solidification direction

As shown in Fig. 5 and Fig. 6, there are pore nucleation, pore interruption, pore coalescence, diameter change of pores and lateral displacement of pores in the actual pore structure. Pore interruption can increase the average interpore spacing and decrease the pore number density. Pore coalescence can increase the average pore diameter and decrease the pore number density. Pore nucleation can decrease the interpore spacing and increase the pore number density. Diameter change of pores and lateral displacement of pores can adjust the diameter and spatial distribution of pores in a local area. These adjustment ways are similar to the elimination and splitting of rods in eutectic growth ${ }^{[26]}$. The elimination of a rod can increase the rod spacing, while the splitting of a rod can decrease the rod spacing.

Similar to the eutectic growth, the coupled growth of gas and solid phases is governed by the interplay between solute diffusion in the liquid and the response of the interfaces due to capillary and kinetic effects. When the interpore spacing is small, the interface energy will increase because of the increasing interface area between solid and gas phases. Thus, pore coalescence or pore interruption will take place which reduces the interface energy. When the interpore spacing is large, the hydrogen concentration will accumulate excessively at the place away from pores. Thus, pores will nucleate in the solute enrichment zone. Through these adjustment ways, the system can return to the stability and the spatial distribution of pores for long distance is uniform.

\section{Conclusions}

An optimized porous copper ingot was fabricated by the Bridgman-type directional solidification method. Evolutions of 
pore structure at different ingot heights were analyzed and 3D pore morphology was observed. Conclusions can be drawn as follows:

(1) As the ingot height increases, the porosity first slightly increases and then remains unchanged. The pore number density decreases, and the decreasing speed of pore number density becomes slower from the ingot height of $85 \mathrm{~mm}$. The average pore diameter increases and then there is a trend of remaining unchanged. The average interpore spacing increases, the increasing speed of average interpore spacing becomes slower from the ingot height of $85 \mathrm{~mm}$. There are no pore clusters and for long distance, the spatial distribution of pores is uniform at different ingot heights.

(2) The evolution of pore structure depends on the change of solidification rate. As the solidification height increases, the solidification rate decreases, and the slope value of solidification rate also decreases. This leads to the change of pore structure.

(3) Pore nucleation, pore interruption, pore coalescence, diameter change of pores and lateral displacement of pores exist in the actual pore structure. The diameter and spatial distribution of pores evolve through these five adjustment ways.

\section{Acknowledgment}

The authors gratefully acknowledge the financial support by the National Natural Science Foundation of China (Grant No. 51371104).

\section{References}

[1] Shapovalov V I. Porous metals. MRS Bulletin, 1994, 19(4): 24-28.

[2] Shapovalov V I. Method for manufacturing porous articles. US patent: 5181549, Jan. 26, 1993.

[3] Nakajima H. Fabrication, properties and application of porous metals with directional pores. Progress in Materials Science, 2007, 52(7): 1091-1173.

[4] Park J S, Hyun S K, Suzuki S, et al. Effect of transference velocity and hydrogen pressure on porosity and pore morphology of lotus-type porous copper fabricated by a continuous casting technique. Acta Materialia, 2007, 55(16): 5646-5654.

[5] Kashihara M, Suzuki S, Kawamura Y, et al. Fabrication of lotus-type porous carbon steel slabs by continuous casting technique in nitrogen atmosphere. Metallurgical and Materials Transactions A, 2010, 41(9): 2377-2382.

[6] Chiba H, Ogushi T, Nakajima H, et al. Heat transfer capacity of lotus-type porous copper heat sink. JSME International Journal, 2004, 47(3): 516-521.

[7] Ogushi T, Chiba H, Nakajima H. Development of lotus-type porous copper heat sink. Materials Transactions, 2006, 47(9): 2240-2247.

[8] Zhang H W, Chen L T, Liu Y, et al. Experimental study on heat transfer performance of lotus-type porous copper heat sink. International Journal of Heat and Mass Transfer, 2013, 56: 172-180.
[9] Liu X B, Li Y X, Zhang H W, et al. Effect of pore structure on heat transfer performance of lotus-type porous copper heat sink. International Journal of Heat and Mass Transfer, 2019, 144: 118641.

[10] Du H, Lu D Z, Qi J Z, et al. Heat dissipation performance of porous copper with elongated cylindrical pores. Journal of Materials Science \& Technology, 2014, 30(9): 934-938.

[11] Liu Y, Li Y X, Wan J, et al. Evaluation of porosity in lotustype porous magnesium fabricated by metal/gas eutectic unidirectional solidification. Materials Science and Engineering: A, 2005, 402: 47-54.

[12] Shapovalov V I. Formation of ordered gas-solid structures via solidification in metal-hydrogen systems. Porous and Cellular Materials for Structural Applications. San Francisco, 1998, 521: 281-290.

[13] Liu X B, Li Y X, Wang J, et al. The pore growth process and pore coalescence process in Gasar copper. Materials Characterization, 2018, 137: 231-243.

[14] Song Q L, Jin Q L, Li Z J, et al. Effects of pore nucleation, growth and solidification mode on pore structure and distribution of lotus type porous copper. Transactions of the Indian Institute of Metals, 2017, 70(6): 1437-1445.

[15] Ide T, Tsunemi A, Nakajima H. Fabrication of porous copper with directional pores by continuous casting technique through thermal decomposition of hydride. Metallurgical and Materials Transactions B, 2014, 45(4): 1418-1424.

[16] Drenchev L, Sobczak J J. Gasars: A specific class of porous materials. Motor Transport Institute, Warasaw, 2009.

[17] Lanzoni L, Radi E, Sevostianov I. Effect of pair coalescence of circular pores on the overall elastic properties. International Journal of Solids and Structures, 2019, 172-173: 38-50.

[18] He Y, Li Y X, Zhang H W, et al. Influence of withdrawing speed on the porous structures of Gasar ingots fabricated by Bridgman method. Journal of Materials Processing Technology, 2017, 245: 106-114.

[19] He Y, Li Y X, Zhang H W, et al. Fabrication of Gasar ingots with straight parallel pores by a Bridgman method. Journal of Materials Processing Technology, 2017, 249: 128-134.

[20] Zhang H W, Li Y X, Liu Y. Gas pressure condition for obtaining uniform lotus-type porous structure by Gasar process. Acta Metallurgical Sinica, 2006, 42(11): 1171-1176. (In Chinese)

[21] Wan J, Li Y X, Liu Y. Spatial distribution of pores in lotus-type porous metal. Journal of Materials Science, 2007, 42(15): 6446-6452.

[22] Romanov V, Lomov S V, Swolfs $Y$, et al. Statistical analysis of real and simulated fibre arrangements in unidirectional composites. Composites Science and Technology, 2013, 87: 126-134.

[23] Jiang G R, Li Y X, Liu Y. Experimental study on the pore structure of directionally solidified porous $\mathrm{Cu}-\mathrm{Mn}$ alloy. Metallurgical and Materials Transactions A, 2010, 41(13): 3405-3411.

[24] Liu Y, Li Y X, Wan J, et al. Metal-gas eutectic growth during unidirectional solidification. Metallurgical and Materials Transactions A, 2006, 37A: 2871-2878.

[25] Ratke L, Alkemper J. Ordering of the fibrous eutectic microstructure of Al-Al3Ni due to accelerated solidification conditions. Acta Materialia, 2000, 48: 1939-1948.

[26] Perrut M, Bottin-Rousseau S, Faivre G, et al. Dynamic instabilities of rod-like eutectic growth patterns: A real-time study. Acta Materialia, 2013, 61: 6802-6808. 